\title{
Tipología de los asentamientos rurales en la Comunidad de Madrid
}

\author{
Carmen Pérez Sierra *
}

\section{INTRODUCCION}

Ante los cambios sociales y económicos experimentados por el medio rural de los paises desarrollados, es muy dificil mantener, a la hora de su estudio, el modelo dicotómico o polar de las sociedades rural y urbana. En efecto, las concepciones tradicionales, heredadas de una época en la que el proceso de urbanización iba asociado a grandes aglomeraciones dispensadoras de bienes y servicios, no explican los procesos sociales que se están operando en los espacios supuestamente rurales cuando se hallan influidos por una ciudad de gran poder urbanizante. Estos ámbitos han visto como sus fomas sociales, y también su estructura económica iban transformándose, al tiempo que se acercaban a los valores, expectativas y estilos de vida considerados arquetípicamente urbanos.

Sistemas de relación, estratificación social, movilidad, heterogeneidad, etc., producen una convergencia entre la ciudad y el mundo rural a medida que éste se transforma como consecuencia del impacto de una ciudad dinámica. Pero medir esas transformaciones tampoco resulta tarea sencilla. Modos de vida tradicionales se entremezclan a nuevos valores, la utilización del suelo no es casi nunca uniforme, y la economía permanece en parte transformada, y en parte ligada a concepciones antiguas, produciéndose un medio de caracter multidimensional extremadamente complejo, que se resiste a clasificaciones simplistas.

* Departamento de Geografía. Universidad Complutense. 
El problema se agudiza al considerar que los instrumentos de medida, dentro de las Ciencias Sociales son ambiguos e imprecisos, y que los resultados difieren sin saber a ciencia cierta si ello se debe a los cambios reales producidos en el objeto medido o a la imperfección del instrumento de medición. Realmente el problema de la medición alcanza también a las ciencias fisicomatemáticas pero la imprecisión y ambigüedad antes aludidas se acentúan en nuestra disciplina, debido sustancialmente a la gran complejidad de la mayor parte de los conceptos utilizados en Geografía Humana. Cierto que Shevky y Bell (1955) con su teoría de Análisis de áreas sociales significaron un avance sustancial, pero la subjetividad subyace en el fondo de esta teoría comprometiendo asi los resultados.

\section{ECOLOGIA FACTORIAL Y TIPOLOGÍA DE LOS ASENTAMIENTOS}

En este sentido, la «ecología factorial» puede resultar una alternativa, pues elimina casi totalmente el elemento subjetivo y apriorístico que podía dirigir la investigación hacia resultados fijados de antemano, subrayando por el contrario de forma aséptica los "factores de la diferenciación del espacio social, y la manera en que éstos se combinan para proyectarse sobre el espacio físico, individualizando así tipos de células ecológicas más o menos homogéneas" (Murdie, R. A., pág. 3)

La «ecologia factorial» no es la única técnica multivariante utilizada por las ciencias sociales: el análisis de conglomerados, de correspondencia, discriminante, etc. intentan también un acercamiento más objetivo a la realidad, pero es el de «mayor prestigio para diferenciar ecológicamente las áreas residenciales en las comunidades urbanas y metropolitanas" (Reed, P. H. pág. 226). Permite, sin apriorismos hipotéticos-deductivos, respetar la mayor parte de la información original, al mismo tiempo que la sintetiza en una serie de dimensiones básicas o supervariables, que dan así respuesta a gran parte de la variación espacial de todas y cada una de las variables analizadas. Aunque el problema se traslada ahora a la hora de elegir las variables, su solución es más fácil si puede obtenerse información exhaustiva y fiable, bajo el supuesto de que existe conexión entre las variables introducidas en el estudio.

Hemos intentado aquí, aplicar el análisis factorial a los municipios menores de 10.000 habitantes de la Comunidad Autónoma de Madrid, para lograr una tipología. En efecto bajo la observación más somera puede apreciarse que la Comunidad madrileña, se encuentra "pertubada" a causa del gran poder urbanizante de la "ciudad regional" en la que se 
ha convertido Madrid con el paso de una economía industrial a una economía de servicios. Medir esa perturbación y sus implicaciones sobre la sociedad rural es lo que vamos a intentar a través de la ecología factorial. $Y$ aunque el trabajo del que partimos es más amplio y ambicioso, aquí vamos a centrarnos en el análisis de los municipios de menos de 10.000 habitantes por si Madrid, y aquellos municipios estadísticamente urbanos introdujesen alguna modificación si se hace un examen conjunto.

Para realizar el análisis factorial se tomaron las 25 variables que figuran en el cuadro 1 a partir de las cuales el análisis demostró que los cuatro primeros factores explicaban el 75,3 por 100 de la varianza total de los datos, tal y como aparece en el cuadro 2.

Tras la rotación de la matriz por el método Varimax, se obtuvo una matriz de saturaciones factoriales con estos cuatro factores y en el cuadro 3 se observa que la comunalidad estimada permite desechar siete variables cuyo coeficiente es bajo, y por lo tanto su poder explicativo escaso. Realizado de nuevo el análisis con las 18 variables restantes se seleccionaron cuatro factores que explican el 85,7 por 100 de la varianza (cuadro 4). En el cuadro 5, se muestran las saturaciones factoriales tras la rotación Varimax, subrayando el máximo valor de cada una de las variables.

Al factor I que explica el 43,8 por 100 de la varianza y se encuentra relacionado significativamente con un nivel de instrucción alta, con el caracter cosmopolita, con el predominio de la población joven, con la escasa población activa agraria, etc., se le denominó "Rango Social" por responder al perfil tópico que los estudios de ecología factorial aplican a este tipo de correlaciones.

Al factor II (17,1 por 100 del total de la varianza), relacionado muy negativamente con la actividad industrial, pero positivamente con la segunda residencia, con fuerte índice de envejecimiento, poco cosmopolitismo y relativamente alejados de Madrid, se le denominó "Segunda Residencia".

El factor III (14,5 por 100 de la varianza) recibió el nombre de "Construcción", por estar altamente relacionado con la renta de la construcción, con el número de obreros en este subsector, y poquísimo con la actividad agraria. Pero resulta poco significativo por la ausencia de saturaciones altas, 10 mismo que el factor IV (10 por 100 de la varianza, alta saturación con la renta de servicios y media con la agraria e industrial) del que se prescindió a la hora de realizar la tipología municipal. 


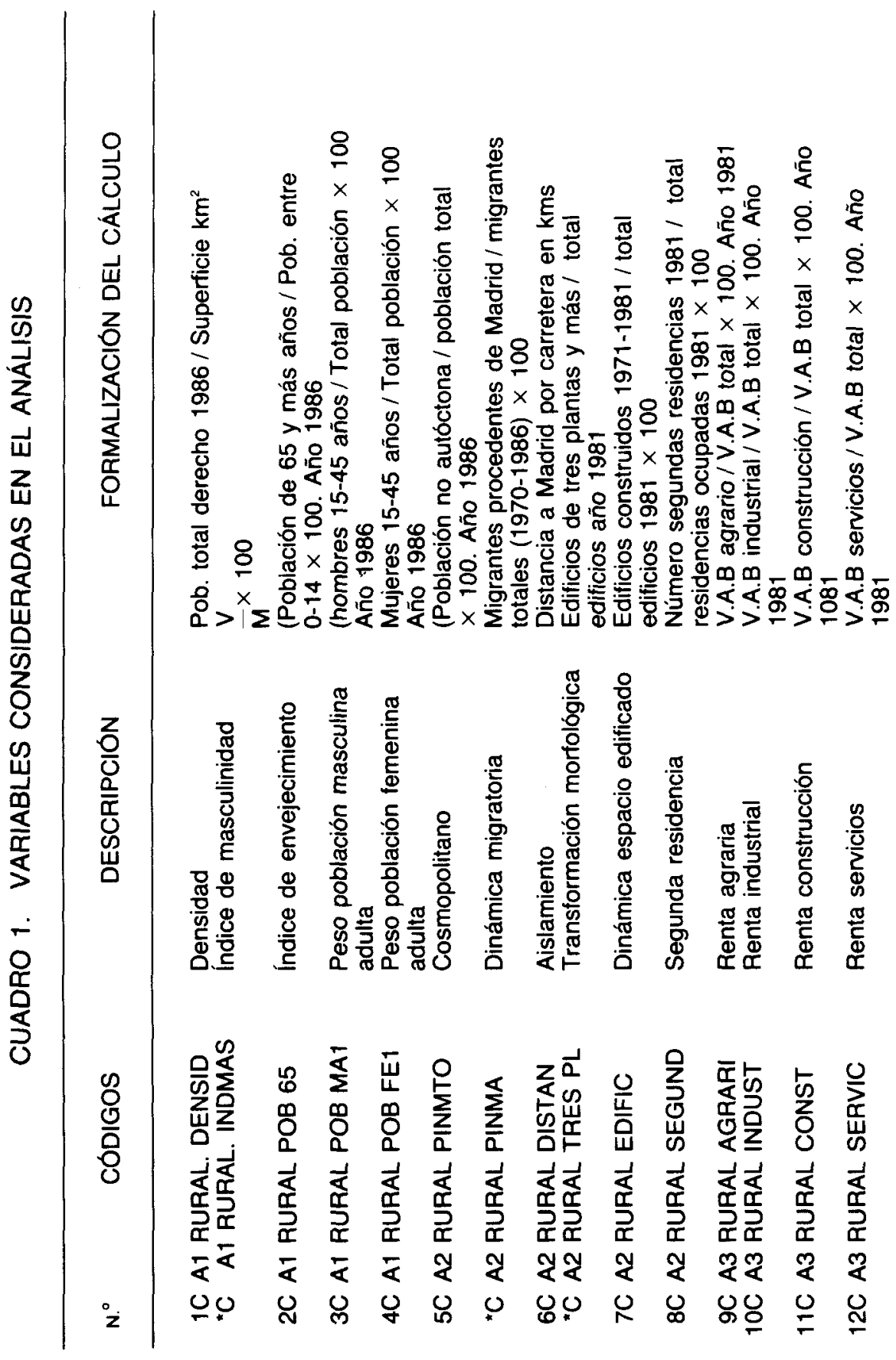




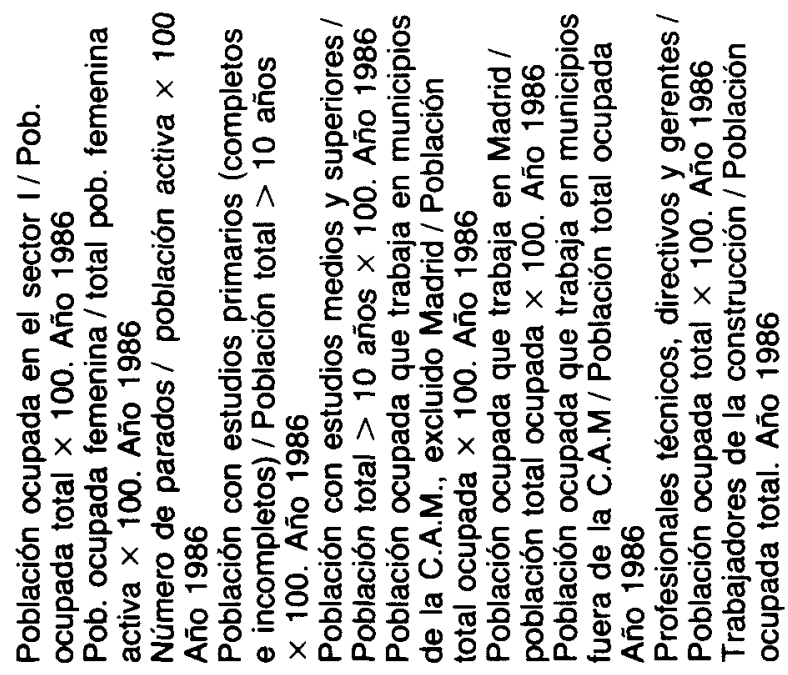

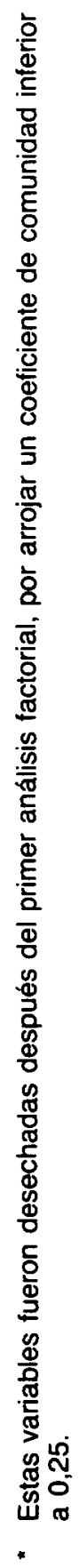

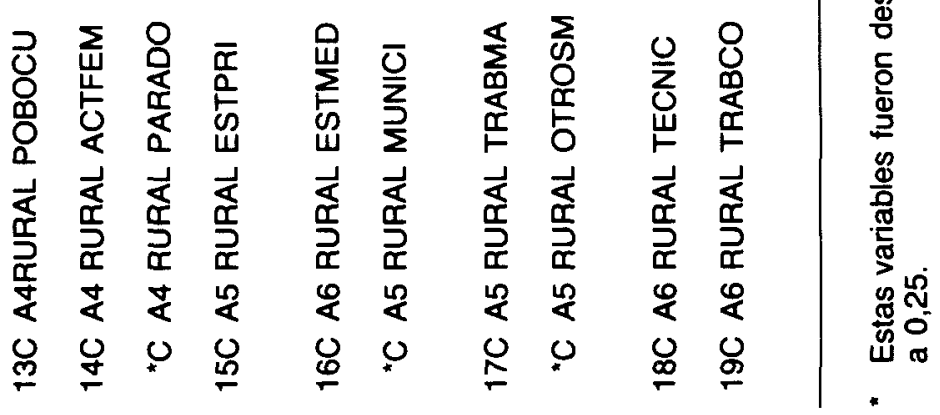


CUADRO 2. RESULTADOS DEL ANÁLISIS DE COMPONENTES PRINCIPALES CON VEINTICINCO VARIABLES

\begin{tabular}{|c|c|c|c|c|c|}
\hline VARIABLE & $\begin{array}{l}\text { COMUNA- } \\
\text { LIDAD }\end{array}$ & FACTOR & $\begin{array}{l}\text { EINGEN- } \\
\text { VALUE }\end{array}$ & $\begin{array}{c}\% \\
\text { VARIANZA } \\
\text { EXPLICADA }\end{array}$ & $\begin{array}{c}\% \\
\text { ACUMULA- } \\
\text { TIVO }\end{array}$ \\
\hline $\begin{array}{l}\text { C:A1RURAL.DENSID } \\
\text { C:A1RURAL.INDMAS } \\
\text { C:A1RURAL.POB65Y. } \\
\text { C:A1RURAL.POBMA1. } \\
\text { C:A1RURAL.POBFE1 } \\
\text { C:A2RURAL.PINMTO } \\
\text { C:A2RURAL.PINMMA } \\
\text { C:A2RURAL.DISTAN } \\
\text { C:A2RURAL.TRESPL } \\
\text { C:A2RURAL.EDIFIC } \\
\text { C:A2RURAL.SEGUND } \\
\text { C:A3RURAL.AGRARI. } \\
\text { C:A3RURAL.INDUST } \\
\text { C:A3RURAL.CONSTR } \\
\text { C:A3RURAL.SERVIC } \\
\text { C:A4RURAL.POBOCU } \\
\text { C:A4RURAL.ACTFEM } \\
\text { C:A4RURAL.PARADO } \\
\text { C:A5RURAL.ESTPRI } \\
\text { C:A5RURAL.ESTMED } \\
\text { C:A5RURAL.MUNICI } \\
\text { C:A5RURAL.TRABMA } \\
\text { C:A5RURAL.OTROSM } \\
\text { C:A6RURAL.TECNIC } \\
\text { C:A6RURAL.TRABCO }\end{array}$ & $\begin{array}{l}0,55426 \\
0,42183 \\
0,75750 \\
0,45267 \\
0,79663 \\
0,71845 \\
0,23001 \\
0,82706 \\
0,26784 \\
0,54392 \\
0,71319 \\
0,98048 \\
0,97897 \\
0,96524 \\
0,98302 \\
0,76156 \\
0,42760 \\
0,31043 \\
0,75834 \\
0,87239 \\
0,34656 \\
0,71352 \\
0,13162 \\
0,86346 \\
0,51208\end{array}$ & $\begin{array}{r}1 \\
2 \\
3 \\
4 \\
5 \\
6 \\
7 \\
8 \\
9 \\
10 \\
11 \\
12 \\
13 \\
14 \\
15 \\
16 \\
17 \\
18 \\
19 \\
20 \\
21 \\
22 \\
23 \\
24 \\
25\end{array}$ & $\begin{array}{r}6,66018 \\
2,65518 \\
1,78931 \\
1,62534 \\
, 93631 \\
, 64149 \\
, 56196 \\
, 43240 \\
, 39204 \\
, 37530 \\
, 30183 \\
, 20399 \\
, 17087 \\
, 11608 \\
, 04294 \\
-, 01675 \\
-, 02412 \\
-, 04446 \\
-, 06194 \\
-, 08533 \\
-, 09885 \\
-, 12076 \\
-, 12784 \\
-, 21140 \\
-, 21512\end{array}$ & $\begin{array}{r}39,4 \\
15,7 \\
10,6 \\
9,6 \\
5,5 \\
3,8 \\
3,3 \\
2,6 \\
2,3 \\
2,2 \\
1,8 \\
1,2 \\
1,0 \\
, 7 \\
, 3 \\
, 0 \\
, 0 \\
, 0 \\
, 0 \\
, 0 \\
, 0 \\
, 0 \\
, 0 \\
.0 \\
, 0\end{array}$ & $\begin{array}{r}39,4 \\
55,1 \\
65,7 \\
75,3 \\
80,8 \\
84,6 \\
88,0 \\
90,5 \\
92,8 \\
95,1 \\
96,8 \\
98,0 \\
99,1 \\
99,7 \\
100,0 \\
100,0 \\
100,0 \\
100,0 \\
100,0 \\
100,0 \\
100,0 \\
100,0 \\
100,0 \\
100,0 \\
100,0\end{array}$ \\
\hline
\end{tabular}


CUADRO 3. ESTIMACIÓN DE LAS COMUNALIDADES CON CUATRO FACTORES

VARIABLE

\begin{tabular}{lll}
$\mathrm{N}^{\circ}$ & COMUNALIDAD \\
ESTIMADA & \\
\hline
\end{tabular}

$\begin{array}{rll}1 & \text { C:A1RURAL.DENSID } & 0,32284 \\ 2 & \text { C:A1RURAL.INDMAS } & 0,18359^{\star} \\ 3 & \text { C:A1RURAL.POB65Y } & 0,66208 \\ 4 & \text { C:A1RURAL.POBMA1 } & 0,31023 \\ 5 & \text { C:A1RURAL.POBFE1 } & 0,66651 \\ 6 & \text { C:A2RURAL.PINMTO } & 0,56941 \\ 7 & \text { C:A2RURAL.PINMMA } & 0,05673^{\star} \\ 8 & \text { C:A2RURAL.DISTAN } & 0,76037 \\ 9 & \text { C:A2RURAL.TRESPL } & 0,07896^{\star} \\ 10 & \text { C:A2RURAL.EDIFIC } & 0,31922 \\ 11 & \text { C:A2RURAL.SEGUND } & 0,56677 \\ 12 & \text { C:A3RURAL.AGRARI } & 0,84865 \\ 13 & \text { C:A3RURAL.INDUST } & 0,73962 \\ 14 & \text { C:A3RURAL.CONSTR } & 0,81720 \\ 15 & \text { C:A3RURAL.SERVIC } & 0,92428 \\ 16 & \text { C:A4RURAL.POBOCU } & 0,62444 \\ 17 & \text { C:A4RURAL.ACTFEM } & 0,20574^{*} \\ 18 & \text { C:A4RURAL.PARADO } & 0,05945^{\star} \\ 19 & \text { C:A5RURAL.ESTPRI } & 0,69403 \\ 20 & \text { C:A5RURAL.ESTMED } & 0,84079 \\ 21 & \text { C:A5RURAL.MUNICI } & 0,24323^{\star} \\ 22 & \text { C:A5RURAL.TRABMA } & 0,64883 \\ 23 & \text { C:A5RURAL.OTROSM } & 0,06803^{\star} \\ 24 & \text { C:A6RURAL.TECNIC } & 0,77163 \\ 25 & \text { C:A6RURAL.TRABCO } & 0,43380 \\ & & \\ & & \end{array}$




\section{CUADRO 4. PROPORCIÓN DE VARIANZA EXPLICADA POR CADA} FACTOR

\begin{tabular}{cccc}
\hline FACTOR & $\begin{array}{c}\text { VALORES PROPIOS } \\
\text { (EIGENVALUES) }\end{array}$ & $\begin{array}{c}\% \\
\text { DE VARIANZA }\end{array}$ & $\begin{array}{c}\% \\
\text { ACUMULATIVO }\end{array}$ \\
& & & \\
1 & 5,89611 & 43,8 & 43,8 \\
2 & 2,30414 & 17,1 & 61,0 \\
3 & 1,95275 & 14,5 & 75,5 \\
4 & 1,36824 & 10,2 & 85,7 \\
5 &, 58239 & 4,3 & 90,0 \\
6 &, 51131 & 3,8 & 93,8 \\
7 &, 30626 & 2,3 & 96,1 \\
8 &, 24960 & 1,9 & 97,9 \\
9 &, 16225 & 1,2 & 99,1 \\
10 &, 07397 &, 5 & 99,7 \\
11 &, 04268 &, 3 & 100,0 \\
12 &,- 00877 &, 0 & 100,0 \\
13 &,- 01477 &, 0 & 100,0 \\
14 &,- 05298 &, 0 & 100,0 \\
15 &,- 10668 &, 0 & 100,0 \\
16 &,- 13728 &, 0 & 100,0 \\
17 &,- 16790 &, 0 & 100,0 \\
18 &,- 18463 &, 0 & 100,0 \\
& & & \\
\hline
\end{tabular}




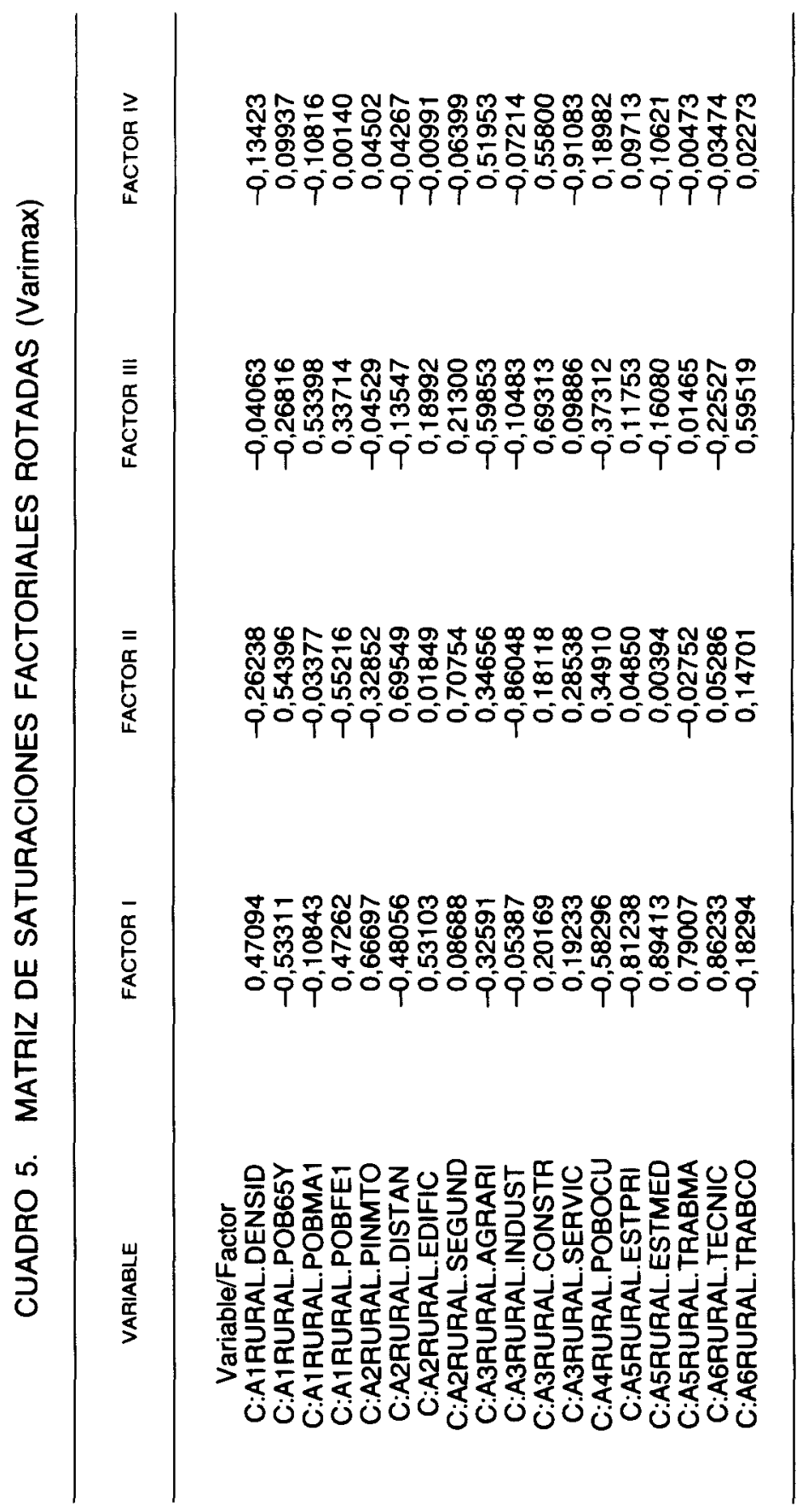




\section{RESULTADOS}

La tipología municipal se estableció teniendo en cuenta la puntuación factorial en cada uno de los municipios considerados.

En el mapa n. ${ }^{\circ} 1$ se cartografían las puntuaciones del factor I. En él puede apreciarse el caracter concentrado del rango social alto en los municipios del noroeste de Madrid. Se trata de aquellos núcleos que fueron elegidos por los madrileños como marco para su segunda residencia en la década de los años sesenta y hoy forman parte de la aureola donde se desar olla el proceso de "suburbanización" que afecta mayoritariamente a personas relativamente jóvenes, pertenecientes a profesiones liberales y con un grado de instrucción alto. En algunos municipios como Los Molinos, Guadarrama, Collado Mediano, Collado de Villalba, Hoyo de Manzanares, compiten con la primera residencia y la segunda y seguramente no tardarán en incorporarse al proceso de suburbanización.

El segundo factor "Segunda Residencia", queda cartografiado en el mapa $n .{ }^{\circ}$ 2. Aparece una fuerte concentración de la segunda residencia en los municipios serranos, especialmente en los municipios de la «Sierra rica" (Guadarrama) en contraposición al sector oriental ("Sierra pobre") donde los valores descienden. En el sector de la Comunidad los valores son bajos, si bien aparecen enclaves con altos valores (Brea de Tajo, Nuevo Baztán, Olmeda de las Fuentes, etc.).

En realidad más de las tres cuartas partes de las segundas residencias se localizan en los municipios de la Sierra, a pesar de que en los municipios occidentales, el fenómeno se encuentra difuminado como consecuencia del citado proceso de suburbanización que convierte las residencias secundarias en principales a medida que mejora el sistema de transportes y aumenta el nivel de vida. Se está configurando por tanto, un espacio especializado como consecuencia de la aparición de un tiempo social, diferenciado por supuesto, según clases sociales.

También en el sector sudoriental aparece un cierto grado de concentración, pero más modesto, porque las externalidades negativas fundamentadas especialmente en la localización industrial, casi ausente en los municipios de la Sierra, y la menor espectacularidad y atractivo del marco natural, determinan una oferta del suelo más barata y por tanto, la ocupación de las segundas residencias por clase media baja, $u$ obreros cualificados, si bien aparecen enclaves con altos valores y además aumento de la calidad: Nuevo Baztán, Olmeda de las Fuentes, etc.

Finalmente, el factor III, "Construcción" aparece cartografiado en el mapa 3. En realidad la interpretación de este mapa no puede efectuarse 

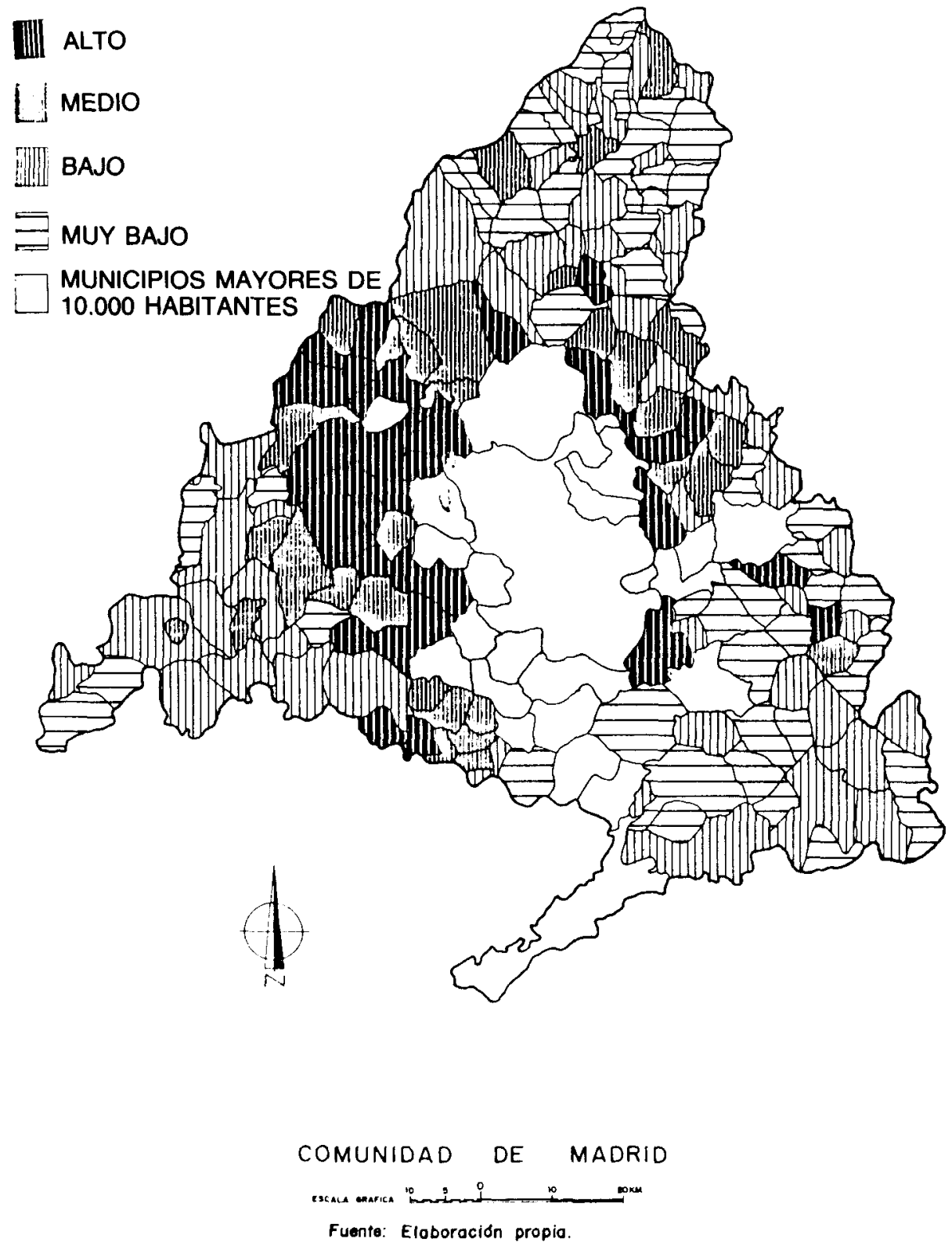

Mapa 1. FACTOR I. RANGO SOCIAL 
ALTO

1. MEDIO

Bin BAJO

펴 MUY BAJO

$\square$ MUNICIPIOS MAYORES DE 10.000 HABITANTES.
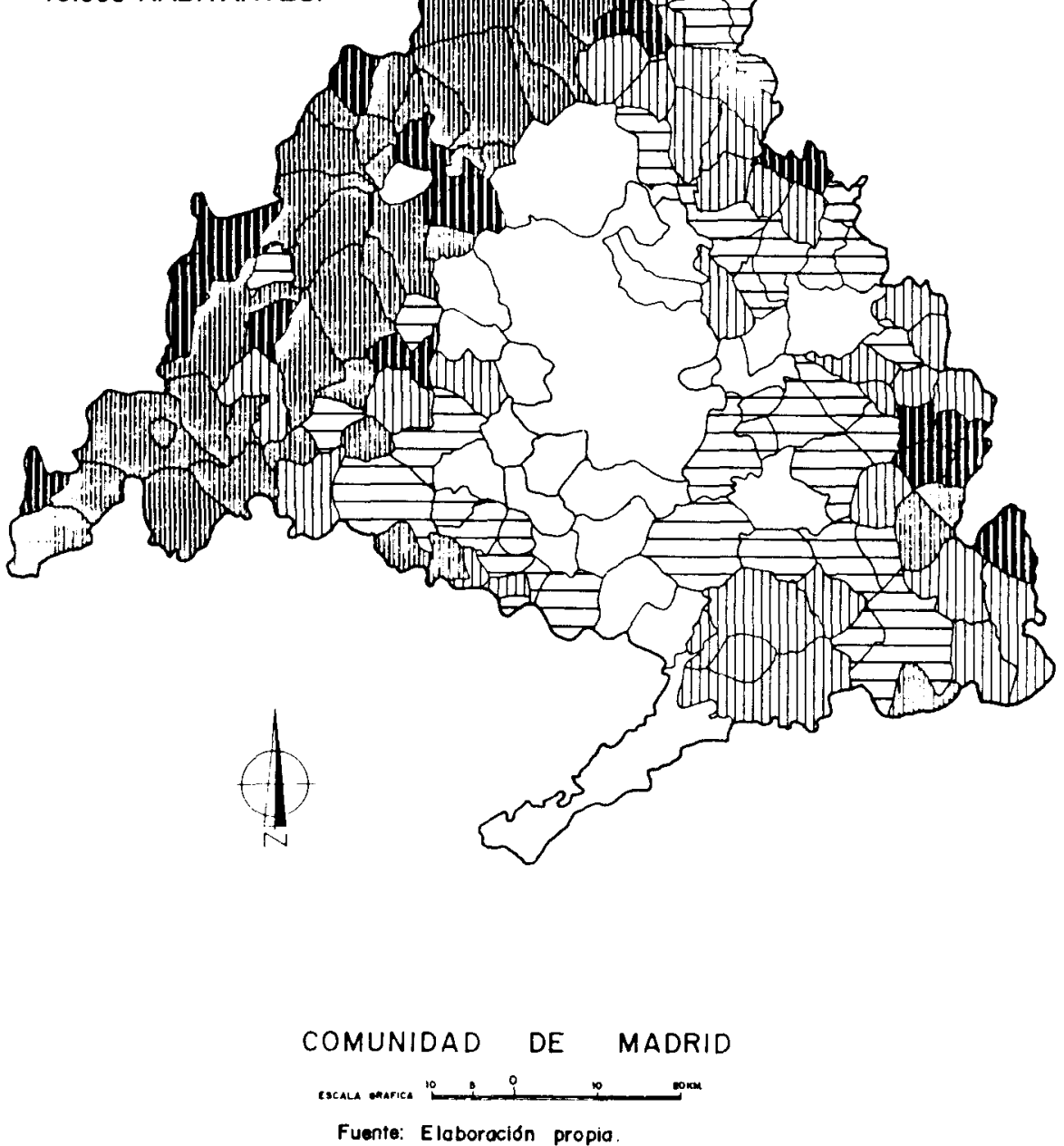

Mapa 2. FACTOR II. SEGUNDA RESIDENCIA 
IIII ALTO

[. MEDIO

Iilll|| BAJO

$\equiv$ MUY BAJO

MUNICIPIOS MAYORES DE

1... 10.000 HABITANTES
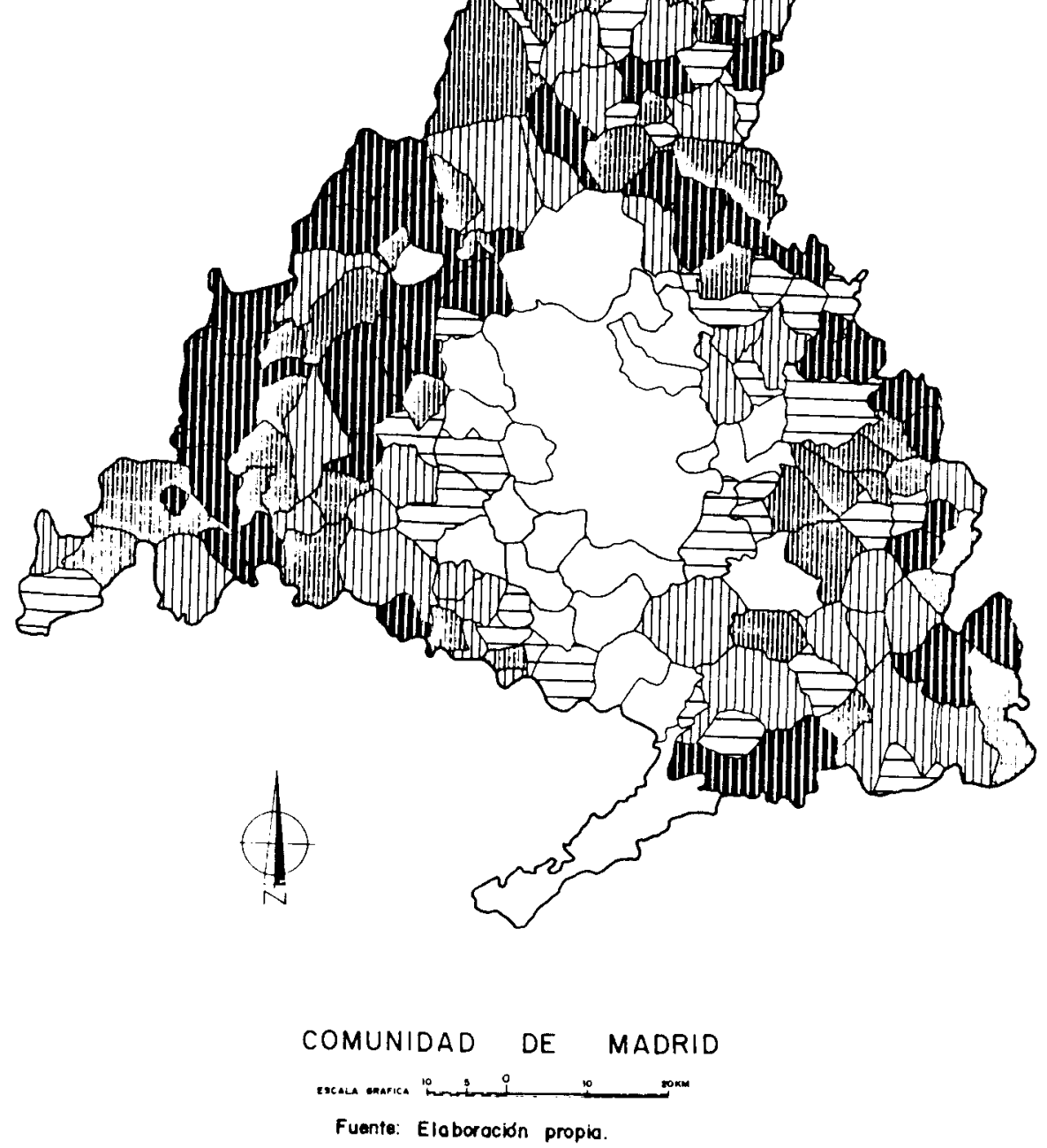

MAPA 3. FACTOR III. CONSTRUCCIÓN 
sin tener en cuenta el anterior, porque en efecto las variaciones en la intensidad de la actividad de la construcción responden indirectamente a la demanda de residencias permanentes o secundarias, pero especialmente de estas últimas en los municipios serranos y en aquellos donde la suburbanización no ha hecho más que empezar. En algunos casos, especialmente en el Sur, y también en las proximidades del Corredor del Henares aparecen valores medios-altos como consecuencia tanto de la segunda residencia como de la descentralización productiva y su consiguiente atracción demográfica.

Sintetizando los tres factores se ha intentado efectuar una tipologia municipal basada en la valoración global de las puntuaciones factoriales en cada uno de los municipios. Para ello se ordenaron los municipios de mayor a menor puntuación en cada factor, y se dividieron en cuartiles dando valor 4 al primero, 3 al segundo, 2 al tercero y 1 al cuarto, sumando en cada municipio la puntuación obtenida. Ningún valor total podia ser mayor de 12, ni menor de 3, puesto que cuatro es el máximo valor alcanzable en cada uno de los tres factores, y 1 el mínimo. $Y$ con esos valores se efectuó una tabla de frecuencias que determinasen los puntos de corte en la distribución, estableciendo cuatro categorias que quedan reflejadas en el cuadro 6, y con las que se elaboró el mapa 4.

Cabe asi destacar una zona de valoración global alta y media, coincidente básicamente en el espacio suburbanizado o de segunda residencia, y en segundo lugar, la franja oriental delimitada claramente en el mapa, con valoraciones bajas y muy bajas, aunque existen enclaves que rompen la uniformidad de este espacio.

Se configura un espacio que responde mayoritariamente a las características de "sombra urbana, umland rural y campo urbano", según el modelo propuesto por Bryant, Ruswürm y McLellan (1982). Pero también una zona de población joven, con fuerte índice de urbanización, altamente cualificada, y ocupada en los subsectores más dinámicos de la economía, que se apoyan en la Sierra o en su peana, y que sigue las líneas de la suburbanización, cada vez más extensa, una vez que las segundas residencias van convirtiéndose en primeras.

El resto del espacio, relativamente urbanizado, se encuentra segregado socialmente; en efecto en el modelo de ciudad regional aparece una fuerte segragación social y funcional del espacio, y en este sentido, en la Comunidad madrileña responde actualmente a la lógica espacial de la salida de la crisis, ya que las empresas industriales y de servicios eligen nuevos emplazamientos, de caracter rural en lo formal, pero con buena accesibilidad, bajo precio del suelo y alto porcentaje de inmigran- 
Tipología de los asentamientos rurales en la Comunidad de Madrid

ALTO

IIIIII MEDIO

BAJO

ఏ MUY BANO

7 MUNCIPIOS MAYORES DE 10.000 HABITANTES
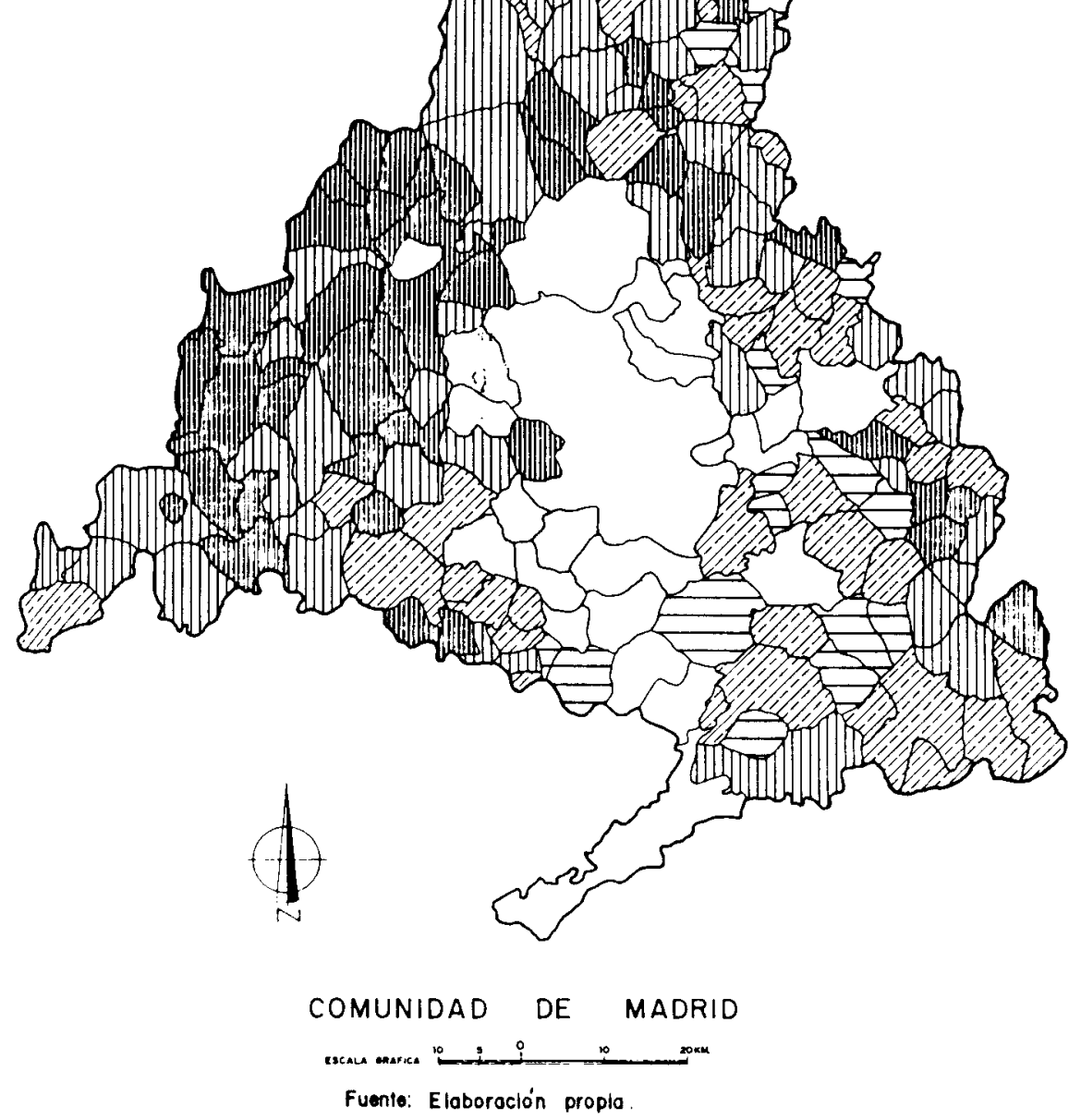

MAPA 4. VALORACION GLOBAL SEGUN LAS PUNTUACIONES DE LOS TRES FACTORES. 


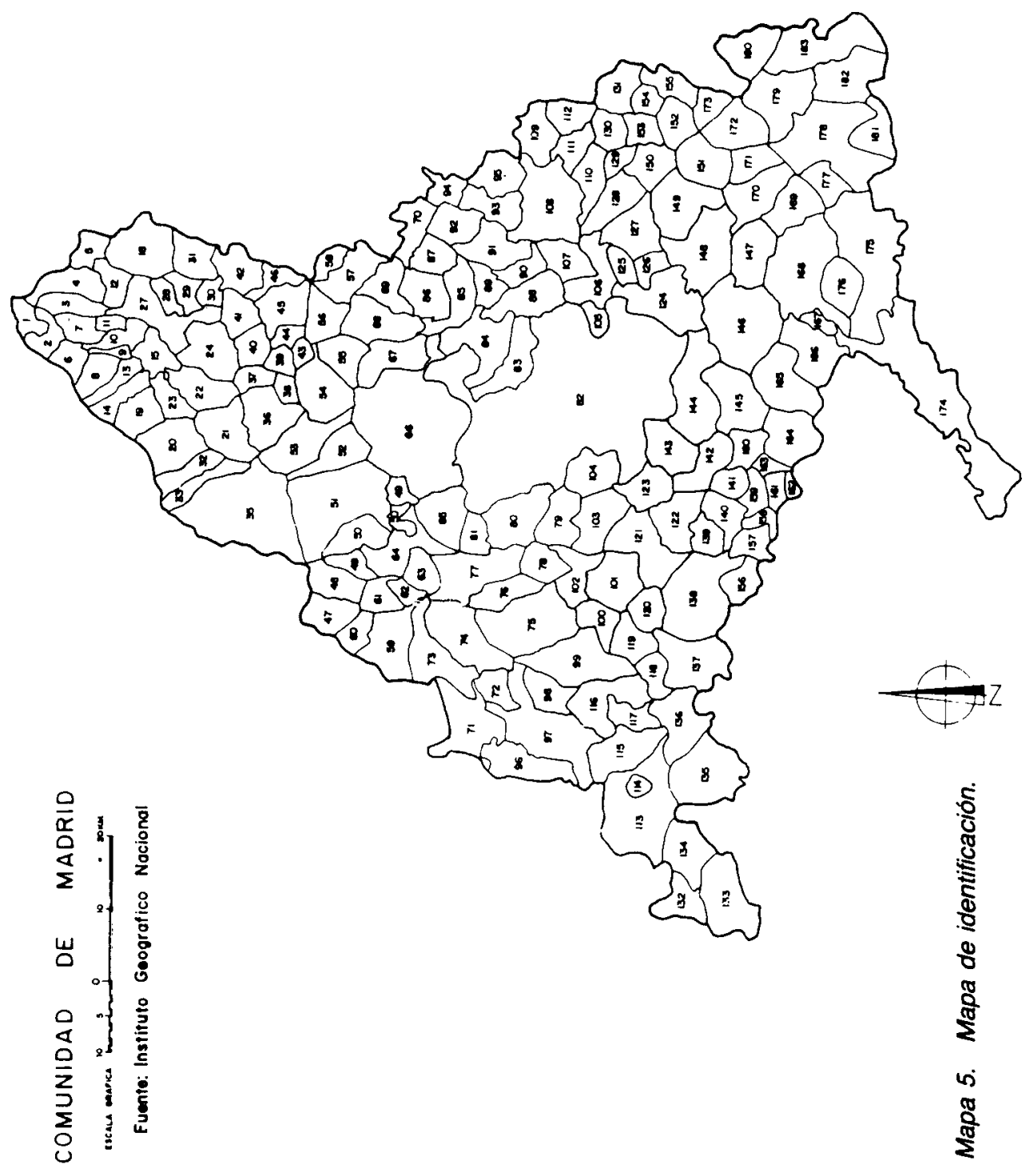




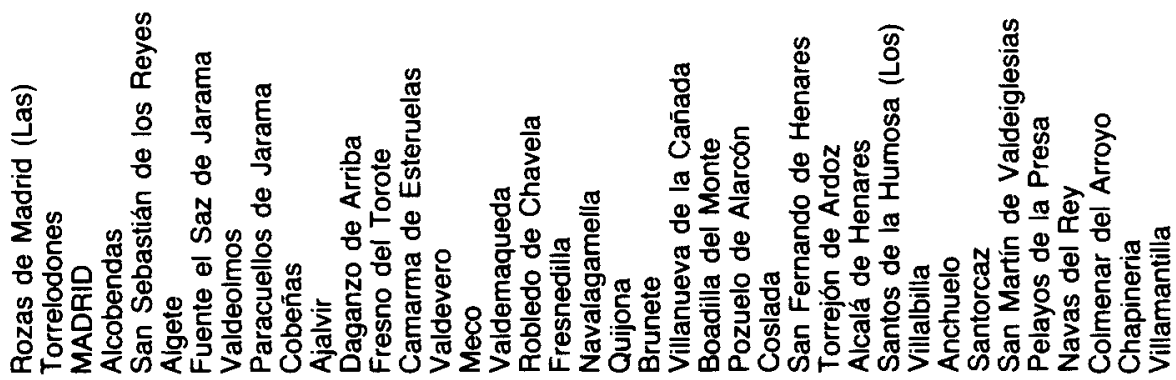

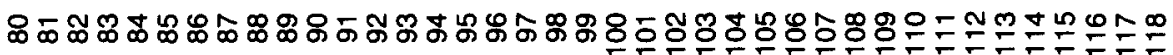

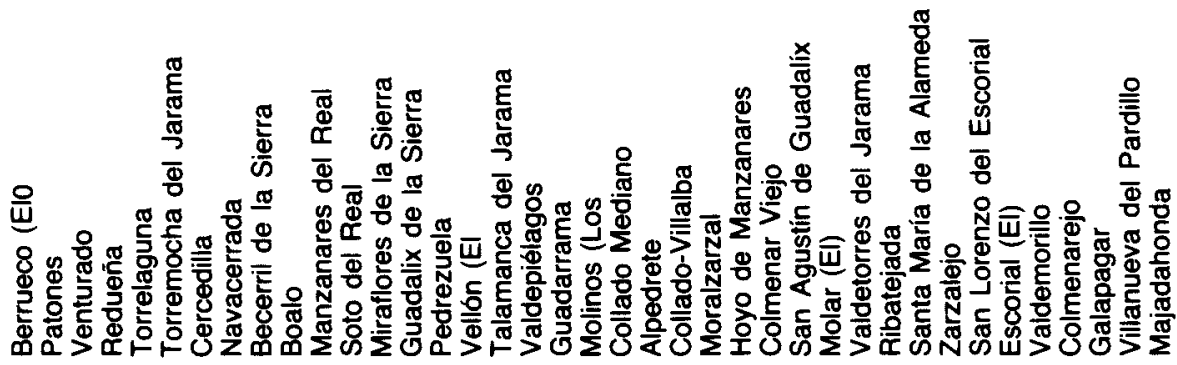

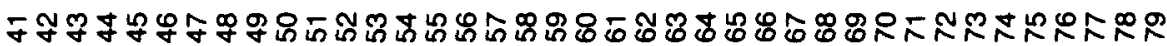

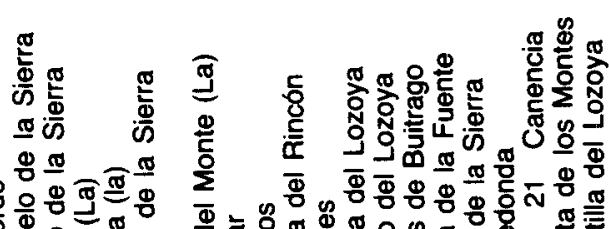

苞

$\frac{\Phi}{\infty}$

约

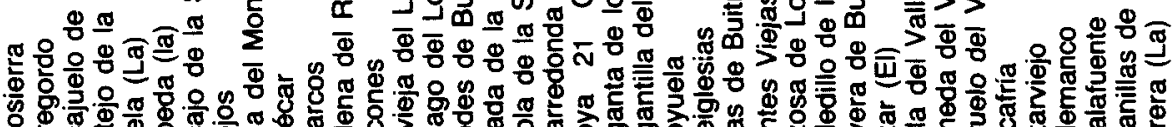

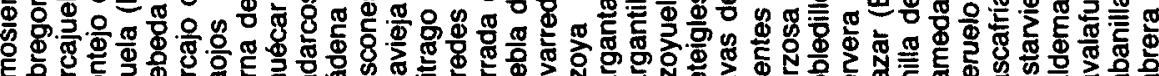

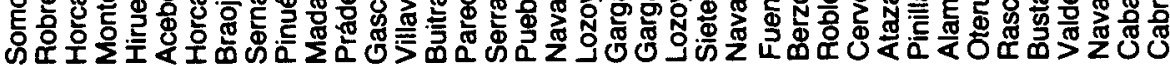

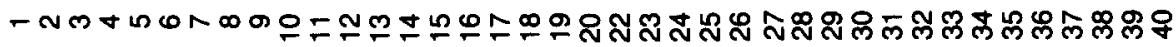




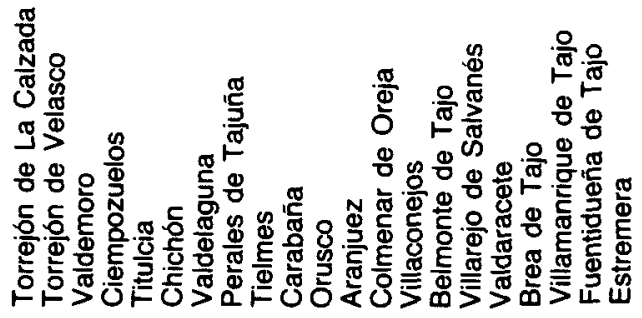

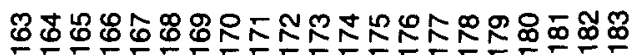
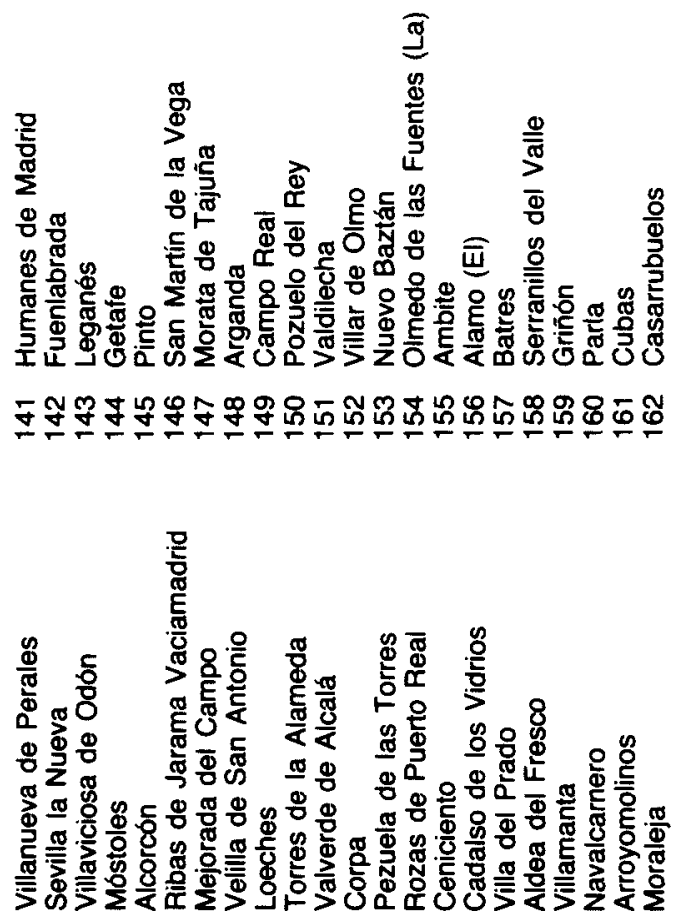

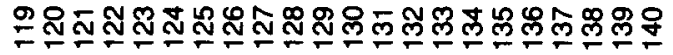


CUADRO 6. CLASIFICACIÓN DE LOS MUNICIPIOS MENORES DE 10.000 HABITANTES

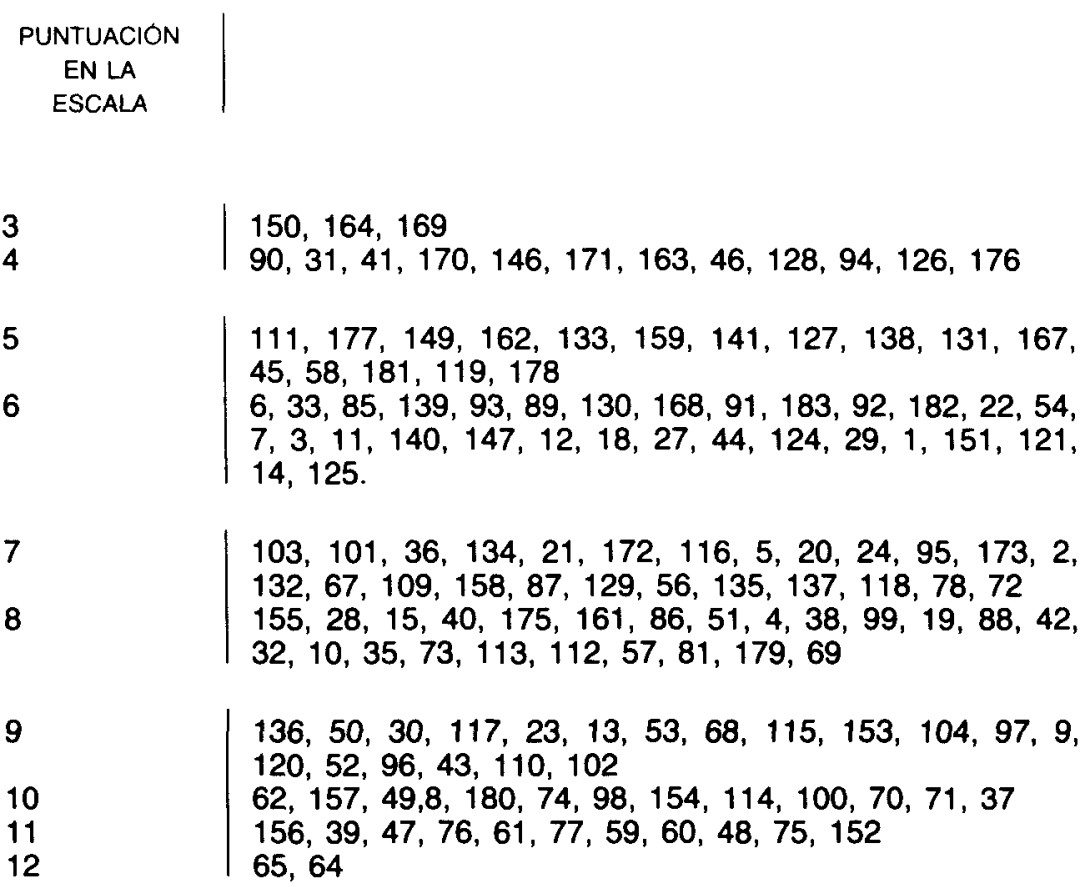

tes escasamente cualificados. Todas estas características determinan un abaratamiento de los costes que atraen la descentralización de las actividades menos productivas de las empresas situadas en la capital o grandes aglomeraciones metropolitanas.

En realidad la ciudad compacta, ha proyectado al convertirse en ciudad regional, la misma segregación social y funcional que en ella se fue formando a lo largo del tiempo, pero ahora en un espacio que se identifica con la casi totalidad de la Comunidad Autónoma madrileña. 


\section{BIBLIOGRAFIA}

Briant, C. R.; Ruswürm, L. H. Y MCLellan, J. (1982): The city's countryside. Land and its management in the rural urban fringe. Londres, Longman. 116.

Murdie, R. A. (1979): The factorial ecology of Toronto. Department of Geography. University of Chicago, Research Paper núm. 116.

REES, P. H. (1975): «Factorial ecology: an extended definition, survey and critique of the field». Economic Geography, vol. 17, núm. 2, págs. 225-236.

SHEVkY, E. y BeLL, W. (1985): Social Area analysis. Stanford University Press. 